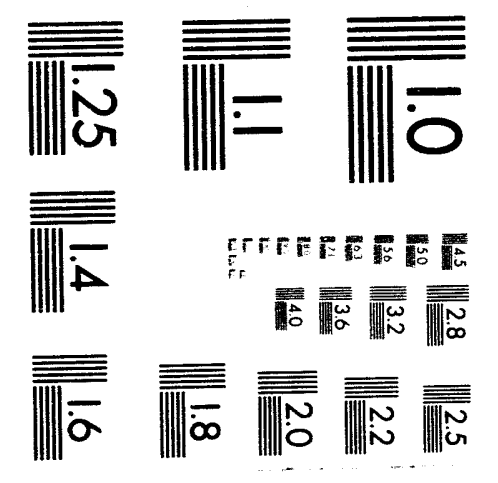





\section{DISCLAIMER}

This report was prepared as an account of work sponsored by an agency of the United States Government. Neither the United States Government nor any agency thereof, nor any of their employees, makes any warranty, express or implied, or assumes any legal liability or responsibility for the accuracy, completeness, or usefulness of any information, apparatus, product, or process disclosed, or represents that its use would not infringe privately owned rights. Referprocess disclosed, or represents that its use would not infringe privately owe name, trademark, ence herein to any specific commercial product, process, or service by trade name, trademark, manufacturer, or otherwise does not necessarily constitute or imply its endorsement, recommendation, or favoring by the United States Government or any agency thereof. The views and opinions fathors expressed herein do not necessarily state or reflect those of the United States Government or any agency thereof.

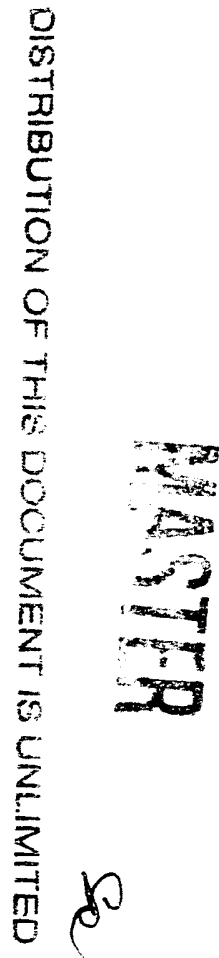

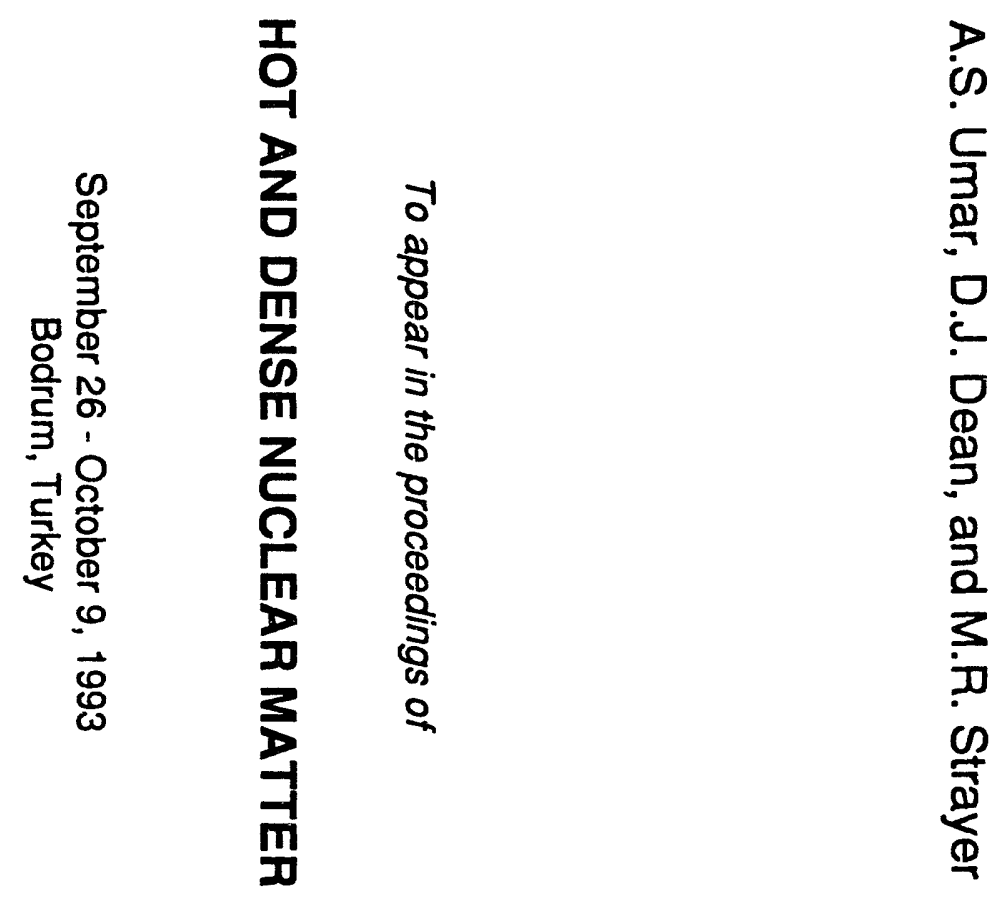

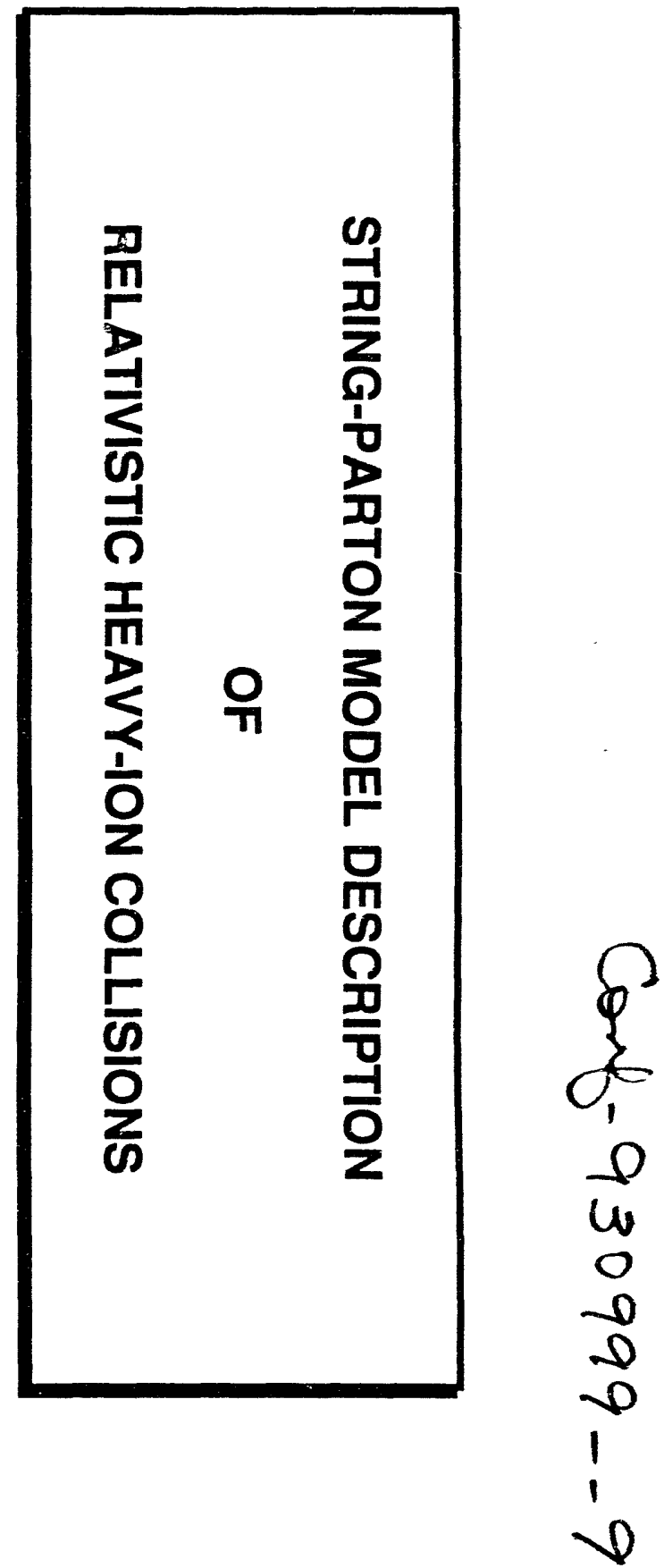




\title{
STRING-PARTON MODEL DESCRIPTION OF RELATIVISTIC HEAVY-ION COLLISIONS
}

\author{
A. S. Umar ${ }^{2,1}$, D. J. Dean ${ }^{3}$, and M. R. Strayer ${ }^{1}$ \\ ${ }^{1}$ Center for Computationally Intensive Physics \\ Physics Division, Oak Ridge National Laboratory, Oak Ridge, \\ TN 37831-6373 \\ ${ }^{2}$ Vanderbilt University, Department of Physics \& Astronomy, \\ Nashville, TN 37235 \\ ${ }^{3}$ W. K. Kellogg Radiation Laboratory, Califoinia Institute of \\ Technology, Pasadena, CA 91125
}

\section{INTRODUCTION}

Various models have been developed to address the ordinary hadronic physics that occurs in relativistic heavy-ion collisions. These include string-based fragmentation models such as the LUND model ${ }^{1}$, and its extensions in FRITIOF ${ }^{2}$, which assume that excited hadrons behave as a chain of color dipoles that move like one-dimensional relativistic strings. Interactions are introduced via multiple small momentum exchanges between the color dipoles of two overlapping strings. Other nondynamical models are the dual-parton model ${ }^{3}$, in which the strings are formed by soft gluon exchange between the valence partons of the colliding hadrons. The quark-gluon string model $^{4}$ (QGSM), also based on the dual parton model, has been developed to study soft parton collisions, and includes rescattering. The strings in the above models are in fact one-dimensional constructions in momentum space, and string evolution is carried out in this space. They are sometimes referred to as the longitudinal phase space models. Any coordinate space quantities that these models may study come from transformations from momentum space one-dimensional string coordinates to configuration space. Relativistic quantum molecular dynamics (RQMD) calculations have also been performed to study relativistic collision phenomen $a^{5}$. This approach combines resonance formation and decay of light hadronic states, and one-dimensional string fragmentation (LUND model) for very heavy resonances. RQMD follows the full space-time evolution of the light hadronic states, and uses one-dimensional mo- 
mentum space evolution for the heavy states via the LUND string description.

We have developed a real-time dynamical model for studying the inclusive properties of hadronic collisions in three-dimensions $6,7,8,9$. The model is based on the Nambu-Goto string description of hadrons supplemented by extensions to incorporate the basic features of the parton model, together with a hadronization mechanism. The advantage of having a fully dynamical model is the possibility to explore the detailed time evolution of the hadronic matter. Furthermore, since the model is fully three dimensional, all of the transverse degrees of freedom are available to the evolving system. The model has been successfully applied to the study of high-energy $e^{+} e^{-}$, $p p^{6}, \mu p$, and $\mu A^{7,8}$ collisions. These calculations have been used to fix all of the parameters present in the model. For all of the calculations presented here, the model parameters have been held fixed at their predetermined values.

\section{STRING-PARTON MODEL ESSENTIALS}

In building the phenomenology of the dynamical string-parton model description of relativistic heavy-ion collisions, it is desirable to start from a description which entails many of the features observed for elementary high-energy processes. One of the most important properties of hadrons is their substructure observed mainly via deepinelastic charged lepton-hadron collisions. In the parton model the scaling behavior is explained in terms of the presence of point-like charged constituents generically called partons. It can be shown in the infinite momentum frame that the scaling variable $x$ is the fraction of the momentum of the nucleon carried by the struck parton. This relation is true only in this frame; however, it is approximately valid in other frames, if the partons are assumed to be massless. Corrections arising from the finite parton mass are usually neglected, as well as the small differences between neutron and proton distribution functions. The string-parton model reproduces the observed structure functions by utilizing the endpoint dynamics of strings; at any given instant the energy and momentum is shared by the string segment (string shrinks or stretches) and the endpoints. This allows the natural identification of these endpoints as partons belonging to the hadron described by the entire string. This identification was first used in the string-parton model to establish a connection between strings and parton structure functions. For the description of baryons, one end represents a single quark whereas the other end a diquark. Each quark carries a baryon number of $1 / 3$ thus giving $B=1$ for baryons. The description of mesons involves a quark at one end, and an antiquark at the other. For relativistic strings at rest, it is natural to define a fractional momentum variable associated with the string endpoints. Assuming collinear motion along the $z$-direction, which will be the boost axis, we define the string longitudinal momentum fraction in terms of the ratio of the light-cone variables,

$$
x_{s}=\frac{k_{0}+k_{3}}{P_{0}+P_{3}}
$$

where $k$ is the endpoint four-momentum and $P$ is the total string four-momentum. The variable $x_{s}$ is Lorentz invariant for boosts in the longitudinal direction. We have shown ${ }^{6}$ that different dynamical states of motion of the strings give rise to different fractional momentum distributions of the string endpoints, which are identified as massless partons. An ensemble average of different dynamical states of the strings 
accurately reproduces the valence quark structure function of the proton 6 .

The initial setup of a nuclear collision is done as follows: nuclei are randomly generated within an impact parameter range by employing a Fermi-density distribution. The nuclear volume is then populated with nucleons, simulated by strings, such that no two nucleons overlap in the initial state. Each nucleon is initialized according to a Monte-Carlo sampling of the strings so as to reproduce the correct parton distribution functions, as described above. The nuclear impact parameter, $b$, may be fixed at a particular value, or given a range, and is distributed as $2 \pi b d b$. The two nuclei are then boosted with the collision $\gamma$, and the time evolution proceeds via the string equations of motion.

In order to simulate hadron-hadron collisions, the strings must interact with each other. This interaction mechanism should lead to excited strings which must then decay via a suitable hadronization mechanism. An assumption is made in the stringparton model, namely that the final state interactions, which confine the partons, act at large space-time distances of the order of the hadron size, much larger than the parton size and the time scale of the current parton interactions. Then, during the time of interaction the parton can be regarded as quasi-free, and the cross section calculated. In the string-parton model all string-string interactions are specified via effective parton-parton scattering and exchange. The probability that an interaction of two strings takes place has the form

$$
W_{A B} \sim \rho_{i / A}\left(x_{A}\right) \rho_{j / B}\left(x_{B}\right) F_{i j}(\vec{b}) \mathcal{P}_{i j}
$$

where $\rho_{i / A}\left(x_{A}\right)$ denotes the probability density of finding a parton of type $i$ with a given parton momentum fraction $x_{A}$ in nucleon $A . F_{i j}$ is the parton impact parameter dependence. $\mathcal{P}_{i j}(\theta)$ denotes the probability for scattering two partons with incoming momentum states $p_{i}$ and $p_{j}$, and outgoing momentum states $p_{1}$ and $p_{2}$, where $\theta$ is the angle between $p_{i}$ and $p_{1}$ measured in the center-of-momentum frame of the partons. For a single parton-parton scattering, this probability has the $\mathrm{t}$ - and $\mathrm{u}$ - channel form as given by first-order perturbative QCD calculations ${ }^{12}$, except here it is treated phenomenologically as

$$
\mathcal{P}_{i j}(\theta)=\mathcal{A} \int_{0}^{\theta} d \theta^{\prime}\left[\frac{s^{2}+u^{2}}{\left(t-m_{t}^{2}\right)^{2}}+\frac{s^{2}+t^{2}}{\left(u-m_{u}^{2}\right)^{2}}\right],
$$

where $\mathcal{A}$ is the normalization such that $\mathcal{P}_{i j}(\pi)=1$. We sample this distribution with Monte-Carlo techniques. We use Monte Carlo techniques to obtain the distribution by randomly choosing $\mathcal{P}_{i j}$ and inverting the equation to find $\theta$. In practice we choose gluon masses $m_{t}=m_{u}=0.25 \mathrm{GeV}$ which give a range of the interaction corresponding to the $30 \mathrm{mb} p p$ inelastic cross section. The effective scattering results in a very different behavior in comparison to the simple Born scattering with zero gluon mass. The effective scattering probability (2) includes the minijet terms in an average fashion. However, minijet phenomena are not expected to be significant at CERN energies. In order to preserve color neutrality, the gluon exchange is followed by a quark exchange ${ }^{13}$. The above interaction mechanism results in excitations of the strings due to the energy-momentum transfer. In nucleus-nucleus collisions all strings are allowed to interact with each other, including the strings produced by fragmentation as described below. In this sense multiple scattering effects are included in the dynamics of the evolution. 
The real-time dynamics of interacting strings must be supplemented by a. hadronization mechanism. Here, the string-parton model utilizes the pair-creation followed by a string breakup method which is similar to flux-tube breaking of the strong-coupled QCD calculations ${ }^{14}$. Some experimental evidence is also provided by the studies of jets in $e^{+} e^{-}$and $p p$ collisions. These experiments demonstrate that jets originate from hard quarks and gluons and provide support that fragmentation takes place within color neutral systems, and not from isolated partons ${ }^{15}$.

In our simulation of high-energy collisions, string-string interaction mechanisins lead to excited strings which stretch and decay by breaking until they reach a predefined minimum mass. Each string may only decay into segments whose masses are above this cutoff mass. The minimum masses are $M_{q}=0.28 \mathrm{GeV}$ for the mesonic strings, and $M_{q q}=0.94 \mathrm{GeV}$ for baryon strings. The cutoff masses are determined by reproducing the correct hadron multiplicities and charge distributions in $e^{+} e^{-}$ collisions ${ }^{6}$. We also note that the cutoff masses are just the minimum and, in practice, a spectrum of final string masses is produced (simulating excited states). The mass spectra obtained in the string-parton model correctly reproduce the observed distributions. The choice for the spatial decay point along the string is based on the invariant area decay law $16,17,6$, in which the probability, $\mathcal{P}$, for a small segment of string to decay is a function of the invariant area it sweeps as it propagates, $\triangle A$, and is given by $\mathcal{P}=1-\exp (-\Lambda \Delta A)$. The decay constant, $\Lambda$, could also be expressed in terms of a proper time interval for decay, $\Lambda=1 / \tau_{0}^{2}$, where $\tau_{0}=0.5 \mathrm{fm} / c$ which was fitted from $\mu p$ collision data ${ }^{7}$.

The pair-creation process is expected, not only to reproduce the longitudinal distributions observed in high-energy collisions, but also to contribute to the transverse momentum distributions. The quark and the antiquark of the created pair could carry equal and opposite nonvanishing transverse momenta. This source of transverse momenta will primarily contribute to the low momentum (approximately $p_{T} \leq 1.0 \mathrm{GeV}$ ) part of the total transverse momentum distribution. In the absence of any fundamental calculations, we choose to parameterize the transverse momentum assignment with a simple exponential distribution function

$$
f\left(p_{T}\right) p_{T} d p_{T} \propto e^{-\alpha p_{T}} p_{T} d p_{T} .
$$

In practice we have used $\alpha=3.88 \mathrm{GeV}^{-1}$ which accurately reproduces transverse momentum distributions in high-energy $p p$ collisions ${ }^{6}$. The created quarks are initially virtual and become on-shell by absorbing energy from the string. The virtual quarks may not interact until they become real; however, other quarks on the string are allowed to scatter as the string propagates. If the source of the transverse momentum is that acquired by the created virtual quarks, this directly influences the time taken by these particles to come on-shell. This can simply be viewed as a manifestation of the Landau-Pomeranchuk effect.

\section{COMPARISON WITH DATA}

We have applied the string-parton model to collision data for $\mathrm{S}+\mathrm{S}, \mathrm{O}+\mathrm{C}, \mathrm{S}+\mathrm{Al}$, and $\mathrm{O}+\mathrm{Au}$ measured at $\mathrm{NA} 35^{11}$ and WA80 ${ }^{10}$ experiments at CERN. The collisions are followed to $100 \mathrm{fm} / c$ in the collision frame, which is a sufficient amount of time for all particle production to have taken place.

The NA35 data which we have addressed is for $\mathrm{S}+\mathrm{S}$ collisions in the impact 
parameter range $0<b<2.5 \mathrm{fm}$ at $200 \mathrm{~A} \mathrm{GeV}^{11}$. In Fig. 1 the rapidity distributions

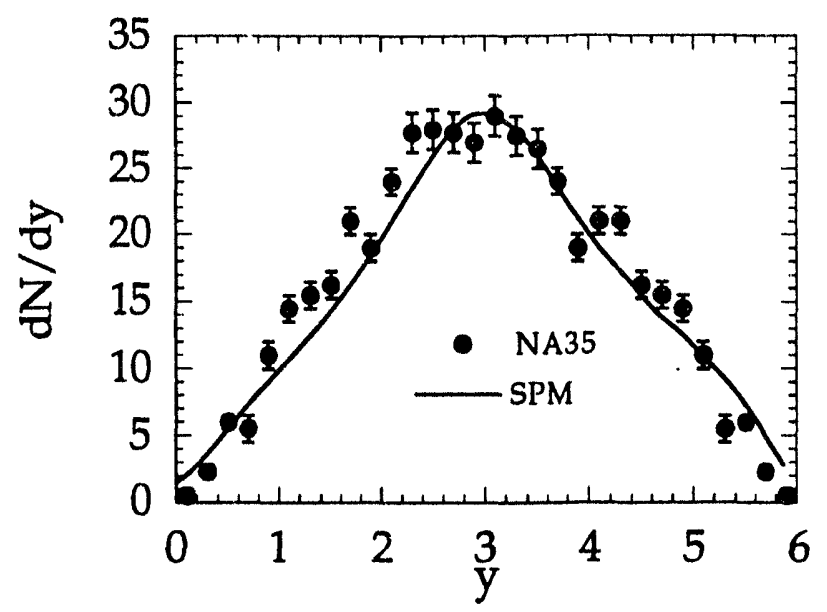

Figure 1: Calculations of the charged pion rapidity distribution for $S+S$ collisions (solid line). Experimental data are from NA35 ${ }^{11}$ (dots). Details of the calculation are given in the text.

are shown for all charged picns, and in Fig. 2 for the charged baryons. The important aspect of this data is that there has been some filling of the central rapidity region, but for the most part the baryons have not stopped. We have shown by calculations that multiple scattering of the baryons and mesons during the collision process is the principal cause for the Gaussian nature of the meson rapidity distributions, and the primary reason for the observed mid-rapidity nucleons.

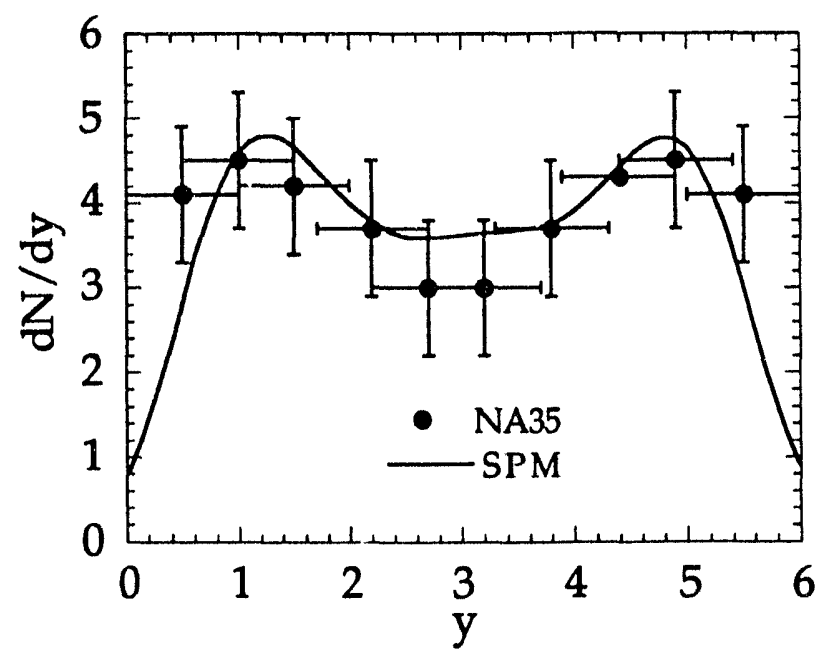

Figure 2: Calculations of the charged baryon rapidity distribution for $\mathrm{S}+\mathrm{S}$ collisions at $200 \mathrm{~A} \mathrm{GeV}$ (solid line) compared to experimental data from NA35 ${ }^{11}$ (dots). Details of the calculation are given in the text.

We have also performed calculations to compare with data from WA80 10 experiments. These experiments consider the transverse energy per unit of pseudo-rapidity produced in asymmetric collisions. We have investigated collisions of $\mathrm{O}+\mathrm{C}$ and $\mathrm{S}+\mathrm{Al}$. We show for the $\mathrm{O}+\mathrm{C}$ and $\mathrm{S}+\mathrm{Al}$ systems the transverse energy spectra in Fig. 3.

All experimental cuts have been imposed on these calculations. In Fig. 4 we show the cross section $d \sigma / d E_{T}$ as a function of $E_{T}$ for the $\mathrm{O}+\mathrm{C}$ and $\mathrm{S}+\mathrm{Al}$ systems. 


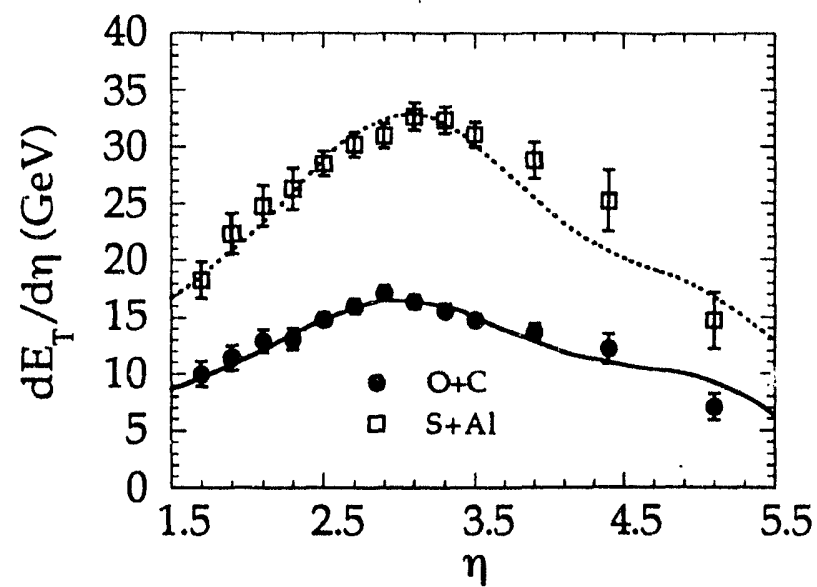

Figure 3: The transverse energy of all hadrons is shown for the $\mathrm{O}+\mathrm{C}$ and $\mathrm{S}+\mathrm{Al}$ systems. Calculations are given by the lines, while experimental WA80 data 10 are shown by the symbols listed on the figure.

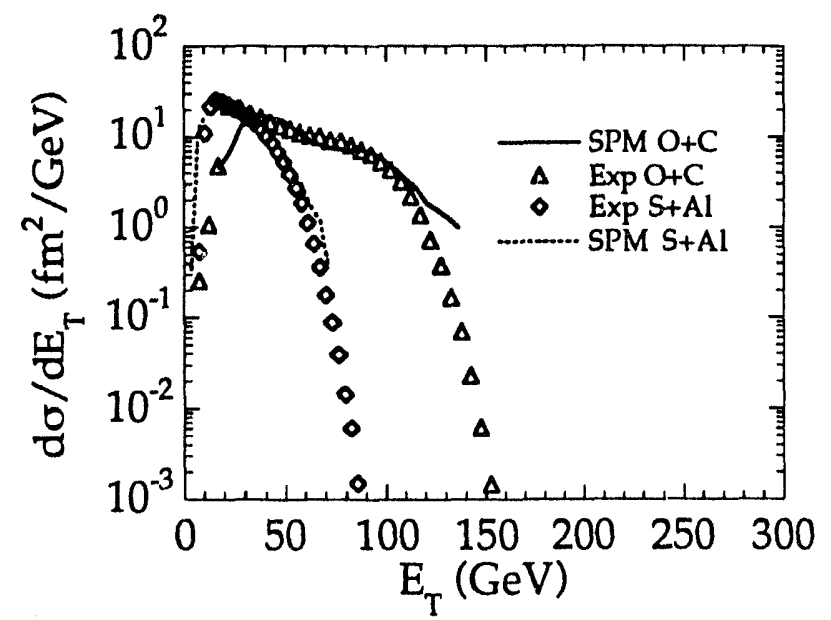

Figure 4: The differential cross section $d \sigma / d E_{T}$ is plotted as a function of $E_{T}$ for the $0+\mathrm{C}$ and $\mathrm{S}+\mathrm{Al}$ systems.

\section{DYNAMICAL ENERGY DENSITIES}

As a fully dynamical theory, the string-parton model can be used to study the time evolution of the meson energy density produced in relativistic nucleus-nucleus collisions. Several procedures may be used to calculate the central energy density. For example, the quark-gluon string model counts all hadrons, including those not yet formed, in energy density calculations ${ }^{4}$. Thus, this calculation obtains significant energy densities for $160 \mathrm{~A} \mathrm{GeV} \mathrm{Pb}+\mathrm{Pb}$ collisions $\left(20 \mathrm{GeV} / \mathrm{fm}^{3}\right.$ at a time of $\mathrm{t}=1.05$ $\mathrm{fm} / c$ ). The VENUS model calculates the energy density with on-shell hadrons, using the "rather arbitrary definition of the hadronization point which is defined as the point where two corresponding [produced] partons meet" for the first time ${ }^{18}$. VENUS calculations obtain $\varepsilon_{\max } \sim 4 \mathrm{GeV} / \mathrm{fm}^{3}$ for $200 \mathrm{~A} \mathrm{GeV} \mathrm{O+Au} \mathrm{collisions.} \mathrm{The} \mathrm{time}$ scale to this maximum density was $\tau \sim 1.0 \mathrm{fm} / c$, which is expected since $\tau \propto \kappa$ in this calculation.

In our calculations of the central energy-densities we will consider only produced on-shell mesons. Although this is in the spirit of the calculations of Ref. ${ }^{18}$, several differences are explained by the different timescales involved in the problem, and of the dynamics of our decay mechanism, as discussed below. Calculations are performed in the center-of-momentum frame of the produced mesons. In this frame the maximal 

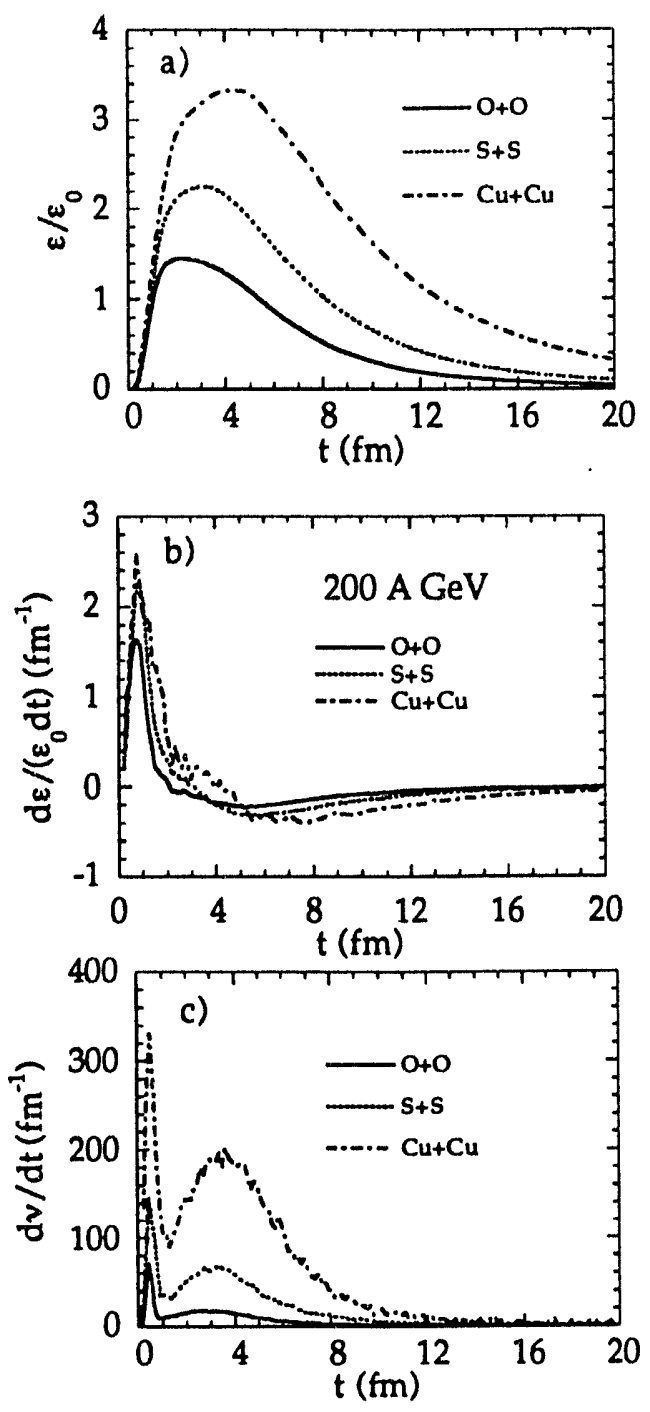

Figure 5: (a) The central meson energy density, defined in the text as a function of time, is plotted for $\mathrm{O}+\mathrm{O}, \mathrm{S}+\mathrm{S}$, and $\mathrm{Cu}+\mathrm{Cu}$ collisions at $200 \mathrm{~A} \mathrm{GeV}$. (b) The time derivative of the energy density is shown for the two systems. (c) The collision rate $d \nu / d t$ is shown for the two systems. In (b) and (c) the derivatives are numerical.

longitudinal extent of the volume, $\ell$, is determined by the separation of the two leading nuclei from the time of their initial contact. The energy and number densities for a radial shell of area $2 \pi r d r$ are then given by $\epsilon(r)=E(r) /(2 \pi r d r \ell)$ and $n(r)=$ $N(r) /(2 \pi r d r \ell)$, where $E(r)$ and $N(r)$ are the energy and number of mesons contained in the shell. The transverse extent of the volume is obtained by sampling the radial meson distribution, $N(r)$. The meson number and encrgy densities at a given time are then

$$
\langle\epsilon\rangle=\frac{\int N(r) \epsilon(r) d^{2} r}{\int N(r) d^{2} r},\langle n\rangle=\frac{\int N(r) n(r) d^{2} r}{\int N(r) d^{2} r} .
$$

The calculation of the energy density is primarily influenced by various time scales involved in hadroproduction. These time scales include the interaction time, i.e. the time taken for the two nuclei to cross each other, the decay or excitation time, and the time required for the created virtual mesons to become on-shell. The model correctly reproduces the experimental transverse energy spectra, shown in the previous section, which is the quantity used in the Bjørken formula for predicting the central energy 
density. For $\mathrm{S}+\mathrm{S}$ collisions the ratio of baryons to mesons in the central rapidity region is on the order of $5 \% 11$. Thus they do not contribute significantly to the measured transverse energy spectrum, $d E_{T} / d \eta$.

In Fig. 5a we show the time dependence of the meson energy density for 200 $\mathrm{A} \mathrm{GeV} \mathrm{O}+\mathrm{O}, \mathrm{S}+\mathrm{S}$, and $\mathrm{Cu}+\mathrm{Cu}$ collisions at zero-impact parameter. We note that the maximum energy density in the central region scales roughly as $A^{0.62}$. Statistical fluctuations in the radial number density, $N(r)$ are on the order of $40 \%$. For the $\mathrm{Cu}+\mathrm{Cu}$ collisions we also considered central slabs of widths $1.0,2.0$, and $3.0 \mathrm{fm}$, and found insignificant differences with the maximum central energy density obtained as described above. We have also checked that slices in rapidity about $y=0$ alter the results by only $10-20 \%$. Inclusion of baryons in the central region, $(|y|<0.5)$, increases the energy density by $10 \%$. We have also made calculations for determining the beam energy dependence of the meson energy density. We find a dependence which roughly scales as $\ln \gamma$. These results predict an approximately $2.9 \mathrm{GeV} / \mathrm{fm}^{3}$ meson energy density for $U+U$ at $100 \mathrm{~A} \mathrm{GeV}$ collider energy. In Fig. 5b we show the time derivative of the scaled meson energy density, $d\left(\varepsilon / \varepsilon_{0}\right) / d t$, where $\varepsilon_{0}=0.16$ $\mathrm{GeV} / \mathrm{fm}^{3}$, which is the energy density in infinite nuclear matter. We also show in Fig. $5 \mathrm{c}$ the collision rate, $d \nu / d t$. An overview of the collision becomes quite clear as we view Fig. 5. For example, for the $\mathrm{S}+\mathrm{S}$ system the two nuclei begin to overlap at $0 \mathrm{fm} / c$, as indicated by the onset of collisions at that time. By approximately 0.5 $\mathrm{fm} / c$, the two nuclei are completely overlapping and particle production has begun, as shown by the $d m / d t$ curve. At $1.0 \mathrm{fm} / c$ the energy production rate has reached its maximum, as indicated in Fig 5b. Secondary collisions of baryons and mesons are indicated by the broad peak of $d \nu / d t$ at $3.0 \mathrm{fm} / c$. The maximum meson mass density appears at roughly the same time as can be seen in Fig. 5a. Note that there are many more secondary collisions in the $\mathrm{S}+\mathrm{S}$ system. These collisions are actually occurring in the baryon-rich regions.

String-parton model calculations of the maximum energy density for mesons formed during nuclear collisions are smaller than the densities given by estimates obtained from the Bjørken formula ${ }^{10}$ by factors of 3 to 4 , depending on the nuclear system involved. This formula is based on the assumption of longitudinal rapidity scaling which is not supported by the data at SPS energies ${ }^{10}$ or by the NA35 data presented above. The Bjørken formula does not take into account the finite size of the nucleus nor the nuclear collision times. Furthermore, the time scale used in the formula is taken to be the strong interaction time, $1 \mathrm{fm} / c$, which does not account for the time required for the virtual particles to become on-shell.

This research was sponsored in part by the U.S. Department of Energy under contract No. DE-AC05-84OR21400 managed by Martin Marietta Energy Systems, Inc., and under contract No. DE-FG05-87ER40376 with Vanderbilt University.

\section{REFERENCES}

1. B. Andersson and G. Gustafson, Z. Phys. C 3, 223 (1980); B. Andersson, G. Gustafson, G. Ingelman, and T. Sjostrand, Phys. Rep. 97, 33 (1983).

2. B. Andersson, G. Gustafson, and B. Nilsson-Almqvist, Nucl. Phys. B281, 289 (1987). 
3. K. Werner, Z. Phys. C 42, 85 (1989).

4. N. S. Amelin, E. F. Staubo, L. P. Csernai, V. D. Toneev, K. K. Gudima, and D. Strottman, Phys. Lett. B261, 352 (1991).

5. H. Sorge, A. v. Keitz, R. Mattiello, H. Stöcker, and W. Greiner, Z. Phys. C. 47 629 (1990).

6. D. J. Dean, A. S. Umar, J. -S. Wu, and M. R. Strayer, Phys. Rev. C 45, 400 (1992).

7. A. S. Umar, D. J. Dean, and M. R. Strayer, in Proceedings of Quark Matter '91, edited by T. C. Awes, F. E. Obenshain, F. Plasil, M. R. Strayer, and C. Y. Wong, Nucl. Phys. A544, 475c (1992).

8. D. J. Dean, M. Gyulassy, B. Müller, E. A. Remler, M. R. Strayer, A. S. Umar, and J.-S. Wu, Phys. Rev. C 46, 2066 (1992).

9. D. J. Dean, A. S. Umar, and M. R. Strayer, Intl. Jour. of Mod. Phys. E.

10. R. Albrecht, et al., WA80 Collaboration, Phys. Rev. C 44, 2736 (1991).

11. H. Ströbele et al., NA35 Collaboration, Nucl. Phys. A525, 59c (1991).

12. R. Cutler and D. Sivers, Phys. Rev. D 17, 196 (1978).

13. N. Isgur, Nucl. Phys. A497, 91c (1989); K. Maltman and N. Isgur, Phys. Rev. D 29, 952 (1984).

14. R. Kokoski and N. Isgur, Phys. Rev. D 35, 907 (1987); G. A. Miller, Phys. Rev. D 37, 2431 (1988); P. Geiger and N. Isgur, Phys. Rev. D 41, 1595 (1990).

15. P. Mättig, Phys. Rep. 177, 141 (1989).

16. K. Sailer, B. Müller, and W. Greiner, J. Mod. Phys. A4, 437 (1989); K. Sailer, B. Müller, and W. Greiner, Proc. of The Nuclear Equation of State, ed. W. Greiner and H. Stöcker, (Plenum, New York, 1990) p.531.

17. E. A. Remler, Proc. of Gross Properties of Nuclei and Nuclear Excitations, Hirschegg, 1987, p.24.

18. K. Werner, Phys. Lett. B219, 111 (1989). 

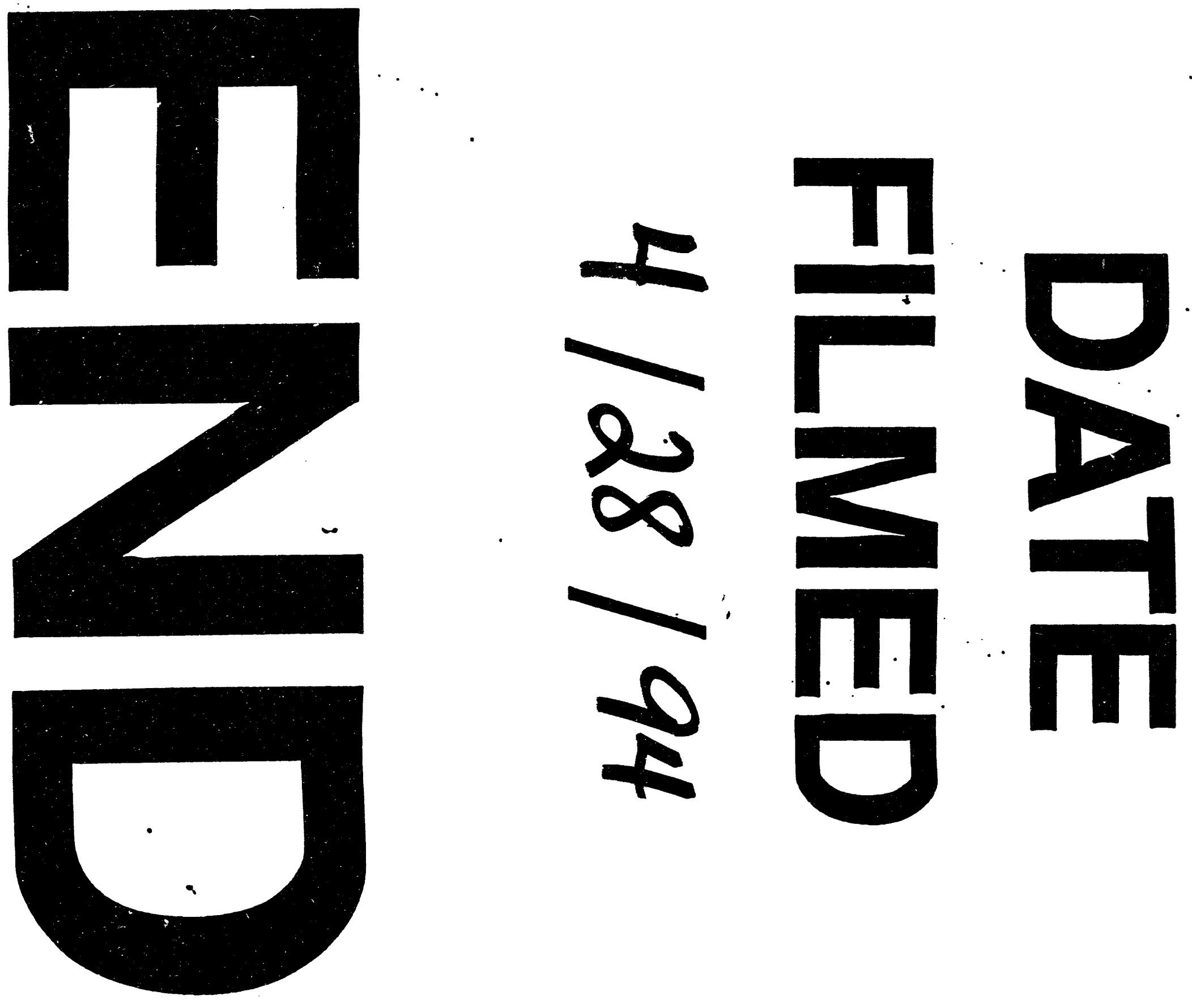